Recepción: 22/01/2019

Aceptación: 29/03/2019

Publicación: 05/04/2019

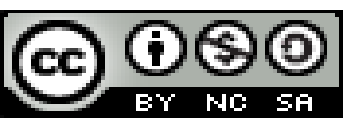

Ciencias sociales y políticas

Artículo de revisión

\title{
Política económica mecanismo de ajuste en la gestión gubernamental
}

Economic policy adjustment mechanism in government management

\section{Mecanismo de ajuste de política econômica na gestão do governo}

\author{
Maryury Elizabeth Morejón-Santistevan ${ }^{\mathrm{I}}$ \\ mayury.morejon@unesum.edu.ec
}

Correspondencia: mayury.morejon@unesum.edu.ec

I Doctora en Administración, Economista, Docente Titular Principal de Universidad Estatal del Sur de Manabí, Manabí, Ecuador. 


\title{
Resumen
}

El presente trabajo se realiza a partir de documentos y escritos inherentes a la política económica, en la que se aprecia el proceso de ajustes y su importancia en la gestión gubernamental a partir de su institucionalización, con la finalidad de alcanzar el bienestar social de los ciudadanos, con la aplicación de la política económica y los instrumentos que la integran, todo esto, como parte componente del gobierno de turno, además debiendo ser conocida por los ciudadanos en la colaboración anticipada con los procesos democráticos; observándose que para alcanzar el bienestar económico debe de existir una interdependencia político-social, que los conduzcan a alcanzar en la población el bienestar requerido desde la actividad económica con la ejecución de políticas públicas, leyes y reglamentos, hasta la regulación por medio de objetivos e instrumentos de la política económica, frente a las fallas de mercado las externalidades, los bienes públicos y el monopolio natural.

Palabras Clave: Política económica; instituciones; instrumentos económicos; bienestar económico; institucionalización.

\begin{abstract}
The present work is carried out from documents and writings inherent to economic policy, in which the process of adjustments and its importance in government management is appreciated from its institutionalization, in order to achieve the social welfare of citizens, with the application of the economic policy and the instruments that integrate it, all this, as a component part of the government of the moment, also having to be known by the citizens in the anticipated collaboration with the democratic processes; observing that in order to achieve economic wellbeing there must be a socio-political interdependence, which will lead them to achieve in the population the well-being required from the economic activity with the execution of public policies, laws and regulations, up to regulation through objectives and instruments of economic policy, in the face of market failures, externalities, public goods and natural monopoly.
\end{abstract}

Keywords: Economic policy; institutions; economic instruments; economic wellbeing; institutionalization. 


\section{Resumo}

Este trabalho é feito a partir de documentos e escrito inerente na política econômica, em que o processo de ajuste e sua importância na governação pode ser visto a partir de sua institucionalização, a fim de alcançar o bem-estar social dos cidadãos com a implementação da política económica e dos instrumentos que integram tudo isso, como um componente do atual governo, ele também deve ser conhecido pelos cidadãos, em cooperação com antecedência com os processos democráticos; observaram que, para alcançar o bem-estar econômico deve ser uma interdependência política e social, o que levou à conquista do bem-estar da população exigido de atividade econômica com a implementação de políticas públicas, leis e regulamentos, a regulamentação por objetivos e instrumentos de política econômica, em face de falhas de mercado, externalidades, bens públicos e monopólio natural.

Palabras Clave: Política econômica; instituições; instrumentos econômicos; bem-estar econômico; institucionalização

\section{Introducción}

En lo concerniente a la Política Económica como mecanismo de ajuste en la gestión del Gobierno, esta se realiza mediante la aplicación de los objetivos e instrumentos que integran la política económica por medio de los gobernantes. Con la puesta en marcha de políticas establecidas por estrategias, para alcanzar el bienestar económico y social de los ciudadanos, desde la institucionalización de las políticas públicas hasta los diversos sectores sociales, logrando una mejora en la distribución del ingreso entre los integrantes de la sociedad.

Por lo consiguiente, Mill (1996) detalla como la eficiencia conduce a las mejoras sociales a través de la actividad útil en la medida que se efectúe. Por lo tanto, siendo proporcional en lo posible al beneficio que se obtiene del trabajo realizado, para lo cual, es oportuno tomar en consideración leyes para los ciudadanos que conduzcan al objetivo de agrandar los esfuerzos de cualquier segmento de la sociedad, evitando la insensibilidad de sus esfuerzos en las diversas actividades, procurando que se presenten desajustes en el desarrollo de los principios fundamentales de la política económica. 
Es oportuno señalar, que el Estado fundamentalmente se presenta en el reconocimiento de una autonomía absoluta y plena en la toma de las decisiones que se puedan presentar, convirtiéndose en varias ocasiones la relación entre el estado y la sociedad, en un ente que está implícito en las practicas del poder y una integración de actores privados, relacionados a las decisiones que los emergen, así el Estado institucionaliza los intereses individuales constituyéndose en el interés incorporado y la expresión neutral de la voluntad.

Siguiendo a Lichtensztejn (2008), señala que en el mercado lo óptimo es maximizar las funciones individuales de utilidad y punto de equilibrio, a partir, de la igualdad de la oferta y la demanda. Tomando en consideración que la competencia perfecta tiene ciertas características que no se desarrollan en su totalidad, como es el caso, de la transparencia en materia de información, en la uniformidad de la negociación de bienes y servicios reconoce a los ofertantes y demandantes como la fuerza del mercado, pero a la vez, no sé acepta que formen agrupaciones de poderío para la determinación de los precios de equilibrios, debido a que los mismos tienen que ser generados a partir de un entorno de competencia perfecta en la que se establezcan acuerdos respectivos.

Sin embargo, en el mercado de bienes, los salarios, divisas y tasas de interés, surgen con cierta semejanza entre el Estado y el mercado en la manera de conducir a la sociedad, como lo detalla Lichtensztejn. A la vez, también se observa una inestabilidad del sistema económico que se presenta en la política, acentuando las asimetrías y refutaciones del desarrollo capitalista. Por consiguiente, se presentan situaciones donde la política económica colabora con la obtención de estabilidades y equilibrios transitorios del sistema económico, con la finalidad de proteger a los sectores sociales débiles y excluidos de las estructuras del poder político.

El propósito de la presente investigación es enfatizar los ajustes que realiza el gobierno, con la finalidad de tener estabilidad económica y social, que conduzcan al bienestar de los ciudadanos por la intervención Estado y Mercado, desde la institucionalización hasta la interdependencia Política y Economía.

Para efectuar el desarrollo del estudio conducente a las conclusiones, se aplicará el método de análisis documental y las técnicas de investigación con entrevistas a los expertos académicos en el área de la temática. 


\section{Política económica: definición objetivos e instrumentos}

La política económica como mecanismo abarca el conjunto de instrumentos, medidas y procedimientos que se utilizan en un sistema político para monitorear el crecimiento económico. Los diversos países establecen determinadas políticas de gobierno, con la finalidad de cumplir sus objetivos, metas y planes estratégicos (Navarrete, 2012), se debe tomar de referencia a la política económica como un plan económico que debe estar inmerso en el mercado internacional, es así, el poder ejecutivo mediante los órganos administrativos y fiscales es el designado para implementar los ajustes económicos y, poder establecer las pautas que deben de seguir.

Sin embargo, notándose instrumentos más enraizados que otros, pero coexistiendo todos como integración del mismo proceso al objetivo principal (el control de la economía), para este caso, el gobierno central, vinculando a la política económica con la puesta en marcha de nuevas empresas en la economía y el empleo como el argumento central del gobierno, debido a que la demanda de empleo es una variable que se presenta en los ciudadanos de manera constante, a la vez estimando contar con oportunidades que el estado debe proveer hacia nuevas posibilidades desde una óptica idealista (Participante 1, entrevista con la autora, 15-10-2018).

Es oportuno, señalar dentro de los objetivos de la Política Económica al crecimiento económico como el encargado de lograr el incremento de las tasas de producción, (Participante 2, entrevista con la autora, 19-10-2018) tomando en consideración el respeto al medio ambiente y, la relación que se establece con la tasa anual del crecimiento del PIB (Producto Interno Bruto) y la tasa de crecimiento del PIB per cápita. Así también, el pleno empleo que garantiza de forma neta la creación de puestos de trabajo contribuye a reducir y prevenir el desempleo, con la estabilidad de los precios, manteniendo una tasa de inflación reducida a través de la medición del IPC (Índice de Precios al Consumidor).

Siguiendo a Cuadrado et al (2006), otro objetivo de la Política Económica es la distribución de la renta y la riqueza, a través de las diferencias que se presentan entre la concentración de la riqueza con el nivel de ingresos personales y el abastecimiento de bienes y servicios públicos, todo esto, determinado por medio del índice de Gini que permite observar la desigualdad que se presenta en 
la renta de las familias correspondientes a las áreas urbanas y rurales en un territorio determinado.

Así también, el equilibrio de la balanza de pagos es otro objetivo importante de la política económica que conduce a reducir el déficit exterior a mediano plazo, manteniendo un nivel adecuado de divisas y liquidez con equilibrio interno hacia el extranjero, por medio del planteamiento de distintas alternativas político-económico, es decir, la que está acorde con la población en el corto y el largo plazo, tomando como base la fundamentación de los objetivos sociales con los económicos (Participante 3, entrevista con la autora, 22-10-2018).

Es oportuno recalcar las consecuencias que se aprecian en la política económica a partir del resultado de la elasticidad de la demanda y la oferta, siguiendo a García (2000) se detecta que para la política económica el tamaño de la elasticidad de la demanda y la oferta tiene resultados que se muestran cuando el gobierno resuelve ubicar un tipo de impuesto a un determinado bien. El productor y el consumidor se podrían ver afectados, dividiéndose entre ellos la atención a la elasticidad de la demanda y oferta. Por tal razón, el gobierno deberá tener presente la referencia de las elasticidades antes de gravar con impuestos a determinados bienes de consumo, con esta consideración se podría mejorar la actuación de la política económica en los ámbitos requeridos.

Por lo tanto, el gobierno se ha caracterizado por aplicar los instrumentos de la política económica que están integrados por (la política monetaria, fiscal, cambiaria de precios y de sector exterior) mostrándose con gran poder discrecional para modificar las reglas de juego dentro de las diversas actividades económicas, conduciendo beneficios para un grupo poblacional y para otros de manera disminuida. Urrunaga et al (2014) especifica que con la distribución y asignación de recursos se regula el funcionamiento económico, donde en varias ocasiones se considera que la regulación es utilizada para la distribución y asignación, que conduce a la asignación eficiente.

En la actualidad, la regulación se desarrolla con un contenido de la economía de mercado, beneficiando la distribución de los recursos hacia la eficiencia social. "La gran complejidad de la política fiscal y en general de la política económica, la política fiscal es una rama que radica precisamente en la multiplicidad de objetivos perseguidos, y por lo tanto es la necesidad de acudir a un número considerable de instrumentos para alcanzar dichos objetivos" (Núñez, 1994:15). 


\section{Características fundamentales y teóricas de la política económica}

Entre las características fundamentales que tiene la política económica, está la habilidad para impulsar el empleo en el largo plazo, convirtiéndose en varias ocasiones en una inhabilidad. Así también, los beneficios de la estabilidad de precios, la importancia de las expectativas y el problema de la inconsistencia temporal, es decir, la estabilización de una variable como puede ser la tasa de intercambio es de suma importancia para obtener un resultado óptimo en la aplicación de la política monetaria como instrumento de la Política Económica. Sin embargo, Mishkin (2007) enfatiza que, a través del abordaje de la estabilización de los precios, se puede llegar a la consolidación monetaria por medio de un abordaje nominal, donde se tome en consideración la debilidad que se puede apreciar por parte del banco central, para ese caso se procede a establecer determinaciones adecuadas para su aplicación en el largo plazo.

Es oportuno, mencionar que para frenar las políticas expansivas de corto plazo que se presentan totalmente refutables con el anclaje nominal, estas pueden exhortar al gobierno a obtener mayor responsabilidad de manera fiscal, actuando a favor de la estabilización de los precios, (Participante 4, entrevista con la autora, 25-10-2018) algunos gobiernos en economías muy poco desarrolladas se han visto sometidos a recurrir al impuesto inflacionario, debido a la ausencia de un anclaje nominal fuerte, produciendo de esa manera mayor inflación al momento de la emisión de dinero por bienes y servicios, dando como resultado la fragilidad en la estabilidad de los precios.

Por lo consiguiente, en el sistema de causación no se descartan el registro que se efectúan de los movimientos de caja, permitiendo identificar los activos y pasivos que presenta el Estado, afectando el patrimonio público para evaluar así los efectos de la actividad gubernamental sobre las variables macroeconómicas reales (Participante 5, entrevista con la autora, 10-11-2019).

Sin embargo, debe aplicarse una metodología que evalúa la aplicación de la política económica a través de gastos, ingresos, superávit y déficit presupuestal; independiente del flujo monetario la ejecución de la partida presupuestal situando en movimiento las variables macroeconómicas, con especial énfasis en la demanda agregada determina que con la misma ejecución presupuestal se puede calcular el ahorro, la producción agregada y el consumo real que integran las cuentas 
nacionales, tal como lo corrobora Hernández (2001) prefiriendo de esta manera a la teoría de los keynesianos en este tipo de análisis metodológico para la constatación y evaluación de la política económica adecuada para ser aplicada en un momento dado.

Una aplicación inicial de la teoría de las expectativas racionales dentro del contexto de la macroeconomía clásica, se presenta frente al análisis de los resultados del output por una expansión monetaria. Siguiendo a Mochón (2006), una nueva opinión de la teoría que conduce a la política económica hacia otra óptica, se enfoca como resultado a partir de una sub escuela que está presente en la macroeconomía clásica denominada "De los ciclos monetarios", en la que se define que la información es asimétrica, aunque se presenten en los mercados la competencia como aquel resultado real de una expansión monetaria sobre el output a corto plazo, así también, en la escuela de las expectativas racionales se muestra que los agentes económicos deben tener presente las políticas que fueron mostradas y dadas a conocer a la población en el momento que se produjeron las famosas promesas de campaña electoral.

Por lo consiguiente, actuando de la mejor manera posible y con integrantes que no produzcan errores sistemáticos, a partir del momento en que se realizan conjeturas de dimensiones económicas (Participante 6, entrevista con la autora, 18-11-2018) a posteriori, cuando la información de los agentes es adecuada, se presenta una diferencia reducida con la finalidad que las autoridades integrantes realicen una política económica adecuada que consolide el entorno económico del país, para lo que fueron realizadas tales adecuaciones, considerando las necesidades de la población conducentes al logro del bienestar.

Cuando los gobiernos son incapaces de implantar compromisos con nuevas políticas, se puede apreciar un inconveniente respecto a futuras políticas, encontrando problemas de inseguridad donde la ciudadanía observa que la política gubernamental establecida no concuerda con la política que fue presentada al inicio de la gestión y, no fue justificada la no aplicación o modificación según se dé el caso (Participante 7, entrevista con la autora, 24-11-2018).

Por lo tanto, la finalidad de que a lo posterior no se den desacuerdos entre los gobernantes y gobernados (Participante 8, entrevista con la autora, 25-11-2018) pueden generar problemas futuros enmarcados a inconvenientes políticos, económicos y sociales, sin embargo la política presentada da la oportunidad de modificaciones a posteriori, pero tomando en consideración lo 
adecuado y, correcto para la población con las necesidades presentadas, las políticas macroeconómicas deben de ser aplicadas hacia el desarrollo poblacional del país.

Siguiendo a Kydland y Prescott (2004), la teoría del control o de optimización dinámica en términos matemáticos, no solamente puede ser analizada a partir de la presente teoría. Sino, que deben ser analizadas como el resultado de un juego donde los que realizan las políticas están integrados por diferentes jugadores, donde cada cual debe estar atento a los resultados que genera, con el objetivo de anticipar las reacciones de los otros jugadores en el contexto actual hacia el futuro.

Así también, en la política fiscal y monetaria, se presenta un escenario con expectativas racionales en equilibrio, en el que se muestra por parte del gobierno una carencia de compromisos hacia una política, muchas veces dando como resultado un bienestar reducido a la ciudadanía con respecto a lo que podría obtener con un gobierno comprometido. "La combinación de medidas monetarias y fiscales se conoce con el nombre de combinación de políticas monetaria y fiscal o simplemente combinación de políticas económicas" (Blanchard y Pérez, 2000:128).

Sin embargo, la teoría moderna de la regulación de servicios públicos define la existencia de monopolio natural cuando el costo de abastecer el mercado se presenta de forma reducida al mostrarse desarrollado por una sola empresa. De esta forma, hay una particularidad tecnológica establecida como subaditividad de costos, que no es otra cosa, que el costo de producir un determinado bien o servicio que el mercado demanda en un momento determinado, resultando igual o menor al costo que se genera en el momento de producir el mismo bien o servicio a través de dos o más empresas, tomando para tal caso la consideración de una empresa que suministra un bien o servicio único y diferente que las otras empresas involucradas (Participante 9, entrevista con la autora, 28-11-2018).

De esta manera, cuando existe la presencia de economías de escala, enseguida se da a conocer la existencia de un monopolio natural, Fiel (1998) determina la condición del monopolio natural tomando en consideración, que no específicamente un monopolio natural va a estar integrado por costos medios decrecientes, sino más bien al presentarse el costo fijo en el momento de 
incrementar una segunda empresa, resultando comparativamente importante al agotarse las economías de escala con el incremento de los costos medios requirentes. Así también, frente a la entrada de empresas competidoras, el monopolio natural debe presentar una sostenibilidad, haciendo conocer su capacidad monopolística de poder seguir conservando su situación cuando se presente la entrada de otras empresas, al no existir o estar establecida alguna prohibición formulada para el ingreso de las mismas.

Es importante mencionar la teoría del utilitarismo, (Participante 10, entrevista con la autora, 3011-2018) la que se presenta arraigada por la descripción de los utilitaristas en general, basa su desarrollo en la supremacía de aquellos placeres que genera la mente sobre los aspectos corporales, en el que se detallan la seguridad, el menor costo posible y como base principal la persistencia, que prevalecen en la naturaleza intrínseca y efectuada, donde se proceden a observar la compatibilidad con el principio de utilidad, caracterizándose por ubicar un mayor valor a ciertos requerimientos presentados en comparación con otros.

Por lo tanto, Mill (1984), establece al bienestar de la sociedad a partir de la representación efectuada como resultado de la sumatoria de utilidades individuales de todos los integrantes de la sociedad, donde cada utilidad está en función de la canasta de consumo de los individuos, relacionada a la vez con el bienestar que debe obtener y generar la aplicación de la política adecuada en base a las necesidades presentadas por la población, que vaya a distribuir los recursos que permitan generar la igualdad esperada de todos los individuos por medio de sus utilidades marginales.

Por lo consiguiente, Rawls (1995) acentúa el desarrollo de una teoría que se muestra contraria a la del utilitarismo, enfatizando así a la teoría rawsaliana (denominada de esta forma en honor a su creador Rawls), basada en el hecho que las personas eligen sus propias decisiones dentro de un contexto de inseguridad, dando como resultado el no poder detectar en qué condiciones podrán presentarse a posteriori en un periodo determinado, de esta forma observándose resultados de cambios en un mismo entorno, varias veces, pudiendo ser acaudalados o sin recursos en situaciones de escasez, donde la sociedad puede mostrarse contraria al riesgo que muestran. Por lo tanto, es conveniente que para la existencia de una sociedad justa se deban considerar ciertos 
esquemas como los servicios básicos, el nivel de libertad, la protección y la seguridad, que provean hacia un desarrollo social.

Notándose que estas dos teorías, el utilitarismo y el rawsalianismo, se contraponen, pero ambas hacen referencia a la consecución esperada del bienestar de la sociedad, todo esto, a través de las diversas actividades que pueda realizar el gobierno de turno para alcanzar el beneficio del conglomerado de la sociedad (Participante 11, entrevista con la autora, 30-11-2018).

\section{La interdependencia político - económica}

Un proceso de modernización en la función pública radica en la estabilidad política y económica, viéndose desarrollada a través una nueva normativa que contenga detallada las debidas reglamentaciones para el fin propuesto. Iacoviello (2014) muestra que las autoridades del servicio civil han ido mejorando de manera mancomunada con la profesionalización y responsabilidad de los directivos a través de los gerentes públicos. Sin embargo, al presentarse la debilidad del desarrollo de políticas que puedan dar como resultado un manejo óptimo y adecuado de normas y reglamentos, se debe de afrontar los nuevos desafíos que pueden surgir para alcanzar la estabilidad político-económica, demostrándose que la interdependencia entre ellas emerge dentro de la gerencia pública institucional, al presentarse de forma adecuada y acertada para la realización de buenos procesos.

Por lo consiguiente, se puede notar la dominación por parte de la ideología socialista, la misma que regía en el programa de investigación denominado economía del bienestar teórico, Buchanan (2005) considera que la identificación de los fallos existentes que podría presentar el mercado se produce a través de la referencia de los esquemas ideales. Así mismo, por medio de la investigación también se daba a conocer que los fallos del mercado que se puedan presentar se deben de corregir con la actuación de los proyectos políticos adecuados.

Por lo tanto, el ideal es llegar a obtener un correcto funcionamiento en el momento que se proceda con la corrección de la falla del mercado con el objetivo principal de su reducción, comparándose a la vez con la actuación correcta que debe presentar la política, donde se proceda a reflejar el desarrollo social con la interacción de la política y la economía hacia el establecimiento de alcanzar el fin determinado. Es decir, el desarrollo económico se relaciona con 
la elaboración de políticas y con la habilidad que se presente en el manejo de las estrategias político - económica, que pueden surgir en un país con el objetivo de conseguir un cambio notable en la economía, con el afán de mejorar la desestabilidad económica y por ende los requerimientos de la sociedad (Participante 12, entrevista con la autora, 05-12-2018).

Lefeber (1985), muestra cómo se da la presencia muchas veces de acuerdos establecidos sin responsabilidad de compromisos hacia situaciones ideológicas, por lo tanto, cuando se trata del nivel de vida de la población se hace mención al desarrollo económico, pero en una economía de mercado para acrecentar el poder adquisitivo de los grupos con ingresos reducidos, debe aumentar la demanda de trabajo y por ende verse reflejado en el aumento del nivel de vida, pudiendo presentarse en el momento que se observa la actividad, sin embargo, no muestra actuación por parte del mercado como institución social a la que representa. No obstante, de manera inmediata y necesaria, debe proceder con la participación y actuación el Estado, es decir, puede manejar los medios de producción con la finalidad de obtener el interés social con el aporte de empresas públicas y privadas, obteniendo en ciertos casos como resultado una economía mixta.

De tal manera, las autoridades económicas en el corto y mediano plazo muestran cuatro objetivos simultáneos: reducir el desempleo, controlar la inflación, la parte fiscal y el manejo del comercio internacional asegurando así un crecimiento sostenido. Martner (2000), señala que la gestión primordial es disminuir las pérdidas sociales por parte de los creadores y realizadores de las políticas, conociendo la inestabilidad posterior y las soluciones que deben adquirirse, todo esto, en ocasiones desarrollado en el largo plazo para aumentar el crecimiento potencial. Así se visiona hacia una inflación disminuida, un moderado gasto público y equilibrio externo, como respuesta a las preferencias establecidas por los consumidores, tomando en consideración los cambios en tecnología que pueden presentar y que ayudan al desarrollo económico y social, se puede determinar que la estructura económica tiende a ir mejorando de manera perseverante hacia los contextos de la economía internacional, observando que una labor significativa en los realizadores de la política económica, son las regularidades entre la parte política y económica. 


\section{Aspectos de la política económica}

La política económica basada en el modelo de desarrollo ISI (Industrialización por Sustitución de Importaciones) empleada en las décadas del cincuenta y sesenta en América Latina, se caracterizó por la sustitución de bienes importados por aquellos que se producían de manera local, indudablemente estas apreciaciones presentaron connotaciones de orden mundial y distributivo y, se daba a conocer si realmente era conveniente que se produjera un crecimiento económico apoyado exclusivamente por las exportaciones (participante 13, entrevista con la autora, 10-12-2018).

Siguiendo a Bertola (2011), cuando se daba un incremento del comercio internacional con precios etéreos y a la vez se generaba la complementación de las materias primas de las industrias exportadoras, los descendientes de los trabajadores del modelo ISI preferían percibir altos salarios, provenientes de la industria y dejar atrás labores de servicios urbanos, que se veían reflejados en el incremento de los obreros muchas veces procedentes de países fronterizos.

Por lo tanto, ciertas políticas pueden perjudicar a un país en su conglomerado, como en las cuotas de importaciones y aranceles potencialmente beneficiosos para los países grandes y en desarrollo, que pueden proceder a determinar la disminución de los precios a nivel mundial, como resultado de la integración que conduce a que se reemplace la producción nacional de alto costo con el resultado del producto de las importaciones. De esta forma se obtendría ganancia para el país, caso contrario, si se producen importaciones fuera de los países integrantes de la línea de importaciones, se obtendría como resultado reducciones en las ganancias del país al importar los productos, es importante considerar estas premisas al momento de efectuar análisis que conduzcan el desarrollo del país (Participante 14, entrevista con la autora, 14-12-2018).

Sin embargo, Krugman y Obstfeld (2006), se contraponen con lo mencionado por Bertola, donde sostenía que el modelo ISI era factible para el bienestar económico de un país, siendo el principal motivo en el momento de comprender las causas de una recesión o expansión económica, sin tomar en consideración la variación de los componentes que integran el gasto para que la política económica propuesta sea la más adecuada. Además, en el análisis respectivo de la renta nacional, se puede detectar el porqué del incremento del PNB (Producto Nacional Bruto) de algunos países 
y la reducción en otros, detectando las causas para tal apreciación, las que son generadas a partir de este agregado macroeconómico importante en la actualidad para determinar el manejo y conducción de la política económica.

De tal manera, cuando las instituciones dentro del entorno analizado muestran debilidades, los costos de transacción pasan a constituirse en costos de relevancia, debido a que pasan a formar parte de la propiedad privada o estatal, es así, que proceden a presentar características correspondientes a la propiedad que pertenecen, Licandro y Licandro (2004) muestran que todo se basa a través de las reglas establecidas en el enfoque presentado, tomando en consideración que la aplicación de la actividad estatal se ve dimensionada hacia la regulación de la parte económica, con sus reglas de aplicación desde el ámbito político, que están determinadas a la vez por la propiedad estatal hasta la consecución del bienestar económico.

Es importante, considerar que el Estado intervenga en la producción de bienes públicos, en la reducción de los problemas públicos y las externalidades negativas, para poder así corregir las fallas del mercado mediante políticas tributarias y de precios, con la finalidad de regular los mercados imperfectos hacia el logro de la eficiencia. Siguiendo a Colomé (2005) señala que al generarse un aumento de los bienes públicos, efectivamente se dará como resultado un incremento del bienestar social que involucra una extensión de los gastos que realiza el estado, a lo posterior se verá reflejado por medio de los ingresos públicos, es importante tomar en consideración esta apreciación en el momento que se genera el aumento de los bienes y gastos públicos, eligiendo las prioridades a considerar y resolver para mejorar el bienestar común de la población.

Por lo general, en una sociedad en democracia debe considerarse que los partidos políticos establezcan y comuniquen cuál será su participación y actuación desde los diversos ámbitos, como es el caso de la parte subsidiaria, así también el componente primordial de aquellos juicios de valor que se pueden presentar hacia la obtención del crecimiento económico, todo esto, con la finalidad de que los ciudadanos conozcan a posteriori, los mejores resultados de los objetivos propuestos para el mejoramiento económico y social de la población (Participante 15, entrevista con la autora, 17-12-2018). 
Es oportuno, destacar la cuantificación de la curva de Phillips donde se puede notar que no se presenta de manera estructural, la misma depende de lo que la ciudadanía espera que el gobierno vaya a realizar. No obstante, dependiendo de un modelo especifico, lo que resulta un conjunto de nuevas propuestas sustentadas y desarrolladas por los seguidores de la nueva macroeconomía clásica, Velasco (1987) demuestra que los mercados se presentan en equilibrio inmediato, debido a los diversos elementos que se exteriorizan solo en aquellos precios que excluyan excesos de demanda y oferta. Por lo concerniente, debido a la presencia anticipada de la política monetaria a lo posterior no afectaría a las variables reales (tasa de interés y el nivel de actividad) debido a su neutralidad en un momento determinado.

Así también, una regla de economía abierta es el tipo de cambio, en este caso, los trabajadores en un país determinado se preocupan por su salario real moderado y sus demandas salariales nominales, pero un gobierno interesado en incrementar la competitividad exclusiva de las exportaciones procederá con la devaluación, la misma que se presenta de forma sorpresiva, inmediata y sin previo anuncio, convirtiéndose en tácticas de la política económica que los conlleva a mostrarse fuertes y competitivos a nivel mundial (Participante 16, entrevista con la autora, 19-12-2018).

De tal manera, la economía neoclásica sostiene que las reformas monetarias y fiscales son la parte focal y fundamental dentro de un proceso de estabilización, muchas veces tornándose difícil las actividades que se presentan entre dos jugadores, como es el caso del gobierno y la ciudadanía, enmarcando situaciones de persuasión y comprensión entre gobernantes y gobernados, pero resultando en varias ocasiones, más complicado realizarlo con los integrantes del sector privado, (Participante 17, entrevista con la autora, 20-12-2018) es así, que deben existir consensos para mejorar las diversas actuaciones de los integrantes del proceso económico.

\section{Política económica y la institucionalidad hacia el bienestar económico-social}

El contacto que surge entre los ciudadanos con el gobierno, inserta a la gestión pública en el seguimiento de la institucionalización. Resultan importantes aquellos requerimientos que surgen de la economía de mercado, así como la sociedad actual denota la significancia de gestión pública. Uvalle (2002), señala que el Estado en concordancia con la administración pública,

\section{0}

Pol. Con. (Edición núm. 32) Vol. 4, No 4, abril 2019, pp. 296-317, ISSN: 2550 - 682X 
responde a las exigencias de las actividades político - económicas, la forma en que se deben de plasmar los objetivos y metas trazadas con sus estrategias institucionales, por tal razón las colaboraciones de una pluralidad de actores muestran el interés adecuado en el procedimiento a la atención y solución de los programas públicos. De tal manera, enfatizan la actuación del gobierno con las habilidades enfocadas a la administración pública, para atender los requerimientos de los ciudadanos hacia la resolución de los problemas públicos presentados.

Sin embargo, para obtener el bienestar económico social óptimo, a través del contexto institucional económico, político y social, se requiere contar con un cimiento legal e institucional conformado por recursos financieros y talento humano, que vayan a permitir su desarrollo más allá de solo definir las políticas adecuadas. Como lo muestra Colino et al (2007), se observa en ciertos países que varios gobiernos no cuentan con una capacidad adecuada que le permitan adquirir decisiones y mantenerlas, coexistiendo una falencia principal para el desarrollo, descubriendo no solo problemas de legitimidad y de eficiencia y, por lo tanto, viéndose reflejados en el incremento del desempleo, la marginación y la pobreza.

De tal manera, todo esto puede llevar a conducir a la inestabilidad de la democracia, en algunos países esto se muestra como resultado de un gobierno que no puede ofrecer bienestar a la ciudadanía, para lo cual se deben tomar decisiones a largo plazo para conservar la conexión con nuevos gobiernos que adquiera el país y que no lleguen a afectar a la ciudadanía, sino a mejorar su desempeño (Participante 17, entrevista con la autora, 18-12-2018).

No obstante, en la trasformación hacia una economía de mercado el Estado se presenta radicalmente distinto, ya que puede estar desarrollado no solo por controles directos, sino por medio del sistema tributario, reglamentaciones básicas y el presupuesto. Todo esto, con la finalidad que se presente un nivel aceptable de ingreso y un sistema tributario equitativo y eficiente. Tanzi (1999) y (2010) sostiene que una disminución de los recursos públicos debe de ajustarse hacia las políticas del gasto, de tal manera, las reglas de juego se establecerían por nuevas reglamentaciones que conlleven a fomentar la competencia. Por lo tanto, los responsables de diseñar la política económica tendrían que procurar un desempeño óptimo hacia el mejoramiento en las posibles estrategias que determine el Estado, a la vez proporcionado por el marco institucional y económico mediante los contextos macroeconómicos actuales. 
Sin embargo, es oportuno señalar, la importancia de las externalidades interinstitucionales, pues deben ser tratadas y reconocidas para obtener un progreso significativo en el sector público. Por lo tanto, no siempre coincide la calidad aplicada de la política económica, hay que diferenciar la calidad de las políticas que aplica un gobierno determinado y la calidad del sector público, la aplicación de la política económica no viene garantizada por la calidad del sector público, debido a que los gobernantes de turno pueden escoger políticas adecuadas hacia el bienestar económico y social, pero lo ideal sería que se dé una semejanza entre la política económica y la calidad del sector público (Participante 18, entrevista con la autora, 19-12-2018).

Así también, los mecanismos de implementación de políticas reconocen los preceptos técnicos para la obtención de un determinado objetivo, denominándose a estos mecanismos como reglas de conducta apropiada, que generen a lo consiguiente el éxito de los resultados esperados en el bienestar económico. Aguilar (1992) muestra que, por lo general, los que manejan aquellos mecanismos, a veces no cuentan con la capacidad de responder a la explicación de que si se llegan a presentar cambios no adecuados a lo estipulado en muchas ocasiones proceden atribuyendo como responsable a las causas externas inadvertidas, de la presencia de los resultados por debajo del nivel óptimo esperado.

Sin embargo, resultando en ciertos casos a través de los modelos de incertidumbre las derivaciones que se vayan a obtener, pueden acogerse a la realidad requirente, aunque las probabilidades demandan de la colaboración de otros procesos que permitan analizar los resultados esperados, todo esto, en la consecución del bienestar económico originado desde los lineamientos institucionales (Participante 19, entrevista con la autora, 20-12- 2018).

De igual manera, los componentes de las finanzas públicas son los estabilizadores automáticos que proceden de tal manera para ajustarse a la actividad desarrollada, como resultado obteniéndose un comportamiento contra cíclico. De Gregorio (2007), enfatiza que los componentes del gasto y del ingreso se incrementan y reducen en ciertos periodos de alta y baja actividad, tomando en consideración estas diversas facetas, para lo cual, se procede a destacar los estabilizadores primordiales como es el caso de los programas sociales sujetos al desempleo con los subsidios integrados.

\section{2}

Pol. Con. (Edición núm. 32) Vol. 4, No 4, abril 2019, pp. 296-317, ISSN: 2550 - 682X 
Por lo consiguiente, en lo que respecta al balance del pleno empleo ajustado cíclicamente y corregido por los efectos cíclicos de los ingresos y gastos, se generan directamente a través del presupuesto público. Por tal razón, se aplican las variables de mediano y largo plazo con el propósito de medir los componentes que integran los impuestos y el gasto, así los estabilizadores automáticos se encontrarían en el nivel óptimo, si se presenta una recesión económica los impuestos efectivos deben ser mínimos que los ingresos estructurales, por lo tanto los impuestos para ser medidos deben presentarse con el producto en pleno empleo, todo esto en miras de obtener el bienestar económico de la sociedad (Participante 20, entrevista con la autora, 21-122018).

Así también, el bienestar social es determinado por medio del bienestar individual de los integrantes de la sociedad según las apreciaciones de la economía normativa, para pronosticar la gestión del gobierno por medio del desarrollo del régimen de las políticas, de tal manera, su determinada evaluación en atención al resultado del bienestar de los individuos participantes, sin embargo, los economistas utilizan el modelo estándar de racionalidad, así con los acuerdos presentados como es el caso de los reglamentos que requieren de la participación de actores hacia la consecución de los objetivos propuestos, todo esto, a partir de los reglamentos constitucionales también se convierte en una parte importante para aquellos economistas constitucionalistas normativos (Participante 21, entrevista con la autora, 22-12-2018).

De tal manera, Vargas (2008) muestra que en ciertas ocasiones la toma de decisiones en la distribución y asignación de recursos públicos, pueden ser presentados de forma restringida desde la capacidad de disposición que muestran a partir del homo economicus y el homo politicus, que no es más, que la interacción entre la economía y la política. Por tal razón las regulaciones que se generen a partir de las diversas actuaciones que contiene cada una hacia las reglas de interacción específica, así como los inconvenientes de información asimétrica que se pueden presentar no permiten el acceso al desarrollo de la misma, todo esto observado desde la óptica del neoinstitucionalismo.

\section{Conclusiones}

Expresando correspondencia con la información documental de escritos, publicaciones y entrevistas realizadas se procede a concluir, que indudablemente se requiere de un trabajo 
mancomunado entre la política y la economía, donde la interdependencia resulta importante, es decir la política requiere de la economía de manera recíproca, desde la institucionalización que los gobiernos de turno realizan con la utilización de los instrumentos que integran la política económica, para mejorar así las actividades económicas hasta el logro del bienestar económico y social de los ciudadanos.

Por lo consiguiente, la aplicación de las políticas públicas, con la pluralidad de actores que colaboren con el interés adecuado en el procedimiento a la solución de los problemas públicos, generan enmiendas a los objetivos económicos y por ende a las estrategias institucionales que aplican los gobiernos en la obtención del bienestar económico, con el afán de crear soluciones a los problemas sociales.

Es así, que el Estado actúa por medio de la regulación económica como mecanismo de ajuste necesario, con el propósito de afrontar las fallas de mercado, la presencia de externalidades y el monopolio natural, para llegar a la solución de problemas de índole económica y, que son solucionadas con ciertas teorías importantes que ayudan a la adquisición de los objetivos propuestos, reduciendo de esa forma las externalidades.

Por consiguiente, en el análisis efectuado a los documentos y entrevista realizadas a expertos, se puede corroborar la presencia de una institucionalización enmarcada con la política económica por medio del Estado, debido que la aplicación de los instrumentos macroeconómicos van direccionados a la obtención del bienestar económico y, dependen de la forma de actuación del gobierno de turno y de la interdependencia que se produzca entre la política y la economía, con la finalidad de alcanzar el bienestar económico-social de la población en un país determinado.

Por tal razón, se aspira para el futuro que se sigan realizando investigaciones en la que se puedan detectar de manera minuciosa el mecanismo de ajuste que aplica la política económica con la participación integral del gobierno, la democracia y la reducción de la asimetría de la información, todos estos, conducidos al bienestar económico del país.

\section{Referencias Bibliográficas}

Aguilar, L. (1992). La Hechura de las Políticas. México. Editorial Miguel Ángel Porrua. 
Bertola, L. y Otros. (2011). Institucionalidad y Desarrollo Económico en América Latina. Chile. Editorial Naciones Unidas CEPAL.

Blanchard, O. y Pérez, D. (2000). Macroeconomía: Teoría y Política Económica con Aplicaciones a América latina. Argentina. Editorial Prentice Hall: Pearson Educación, p. 128 .

Buchanan, J. (2005). "Elección Pública: Génesis y Desarrollo de un Programa de Investigación” Revista Asturiana de Economía. 33 (2) pp. 203 -221.

Colino, C y Otros (2007). Avances y Obstáculos en el Fortalecimiento del Estado en Centroamérica y República Dominicana: Un Análisis de la Capacidad Institucional, la Reforma de la Administración y la Gestión Pública. España. Editorial INAP.

Colomé, R. (2005). Microeconomía. Principios y Políticas. Argentina. Editorial Universidad Nacional de Córdoba.

Cuadrado, J y Otros. (2006). Política Económica Objetivos e Instrumentos, Tercera Edición España. Editorial Mc Graw Gill.

De Gregorio, J. (2007). Macroeconomía Teoría y Políticas. 1ra. Edición. Chile. Editorial Person.

Fiel. (1998)La Regulación de la Competencia y de los Servicios Públicos Teoría y Experiencia Argentina Reciente. Argentina. Editorial Fundación de Investigaciones Económicas Latinoamericanas.

García, V. (2000). Para Entender la Economía Política y la Política Económica. México Editorial Centro de Estudios Monetarios Latinoamericanos.

Hernández, I. (2001) "El Proceso Presupuestal Colombiano: Comentarios a la Propuesta de la Misión Alesina”. Revista de Economía Institucional 5(2) pp. 228-245.

Iacoviello, M. (2014) Diagnóstico institucional del servicio civil en América Latina: Ecuador. Banco Interamericano de Desarrollo Disponible: https://publications.iadb.org/handle/11319/6625?locale-attribute=es.(Consultado 25/10/2018). 
Krugman, P. y Obstfeld, M. (2006). Economía Internacional Teoría y Política, Séptima Edición. España. Editorial Pearson Addison Wesley.

Kydland, F, y Prescott, E. (2004). "Las contribuciones de Finn Kydland y Edward Prescott a la macroeconomía dinámica: la consistencia temporal de la política económica y las fuerzas motrices detrás de los ciclos económicos”. Cuestiones Económicas.20 (2). pp. 121 - 164.

Lefeber, L. (1985). La Economía Política del Ecuador Campo, Región, Nación. Ecuador. Editorial Porvenir.

Licandro, G. y Licandro, J. (2004). Una Agenda de Reformas para el Sistema Financiero Uruguayo. Uruguay. Editorial Upeace - Cmip.

Lichtensztejn, S. (2008). Enfoques y Categorías de la Política Económica. México. Editorial Universidad Veracruzana.

Martner, R. (2000). Política Fiscal y Entorno Macroeconómico. Chile. Editorial Naciones Unidas.

Mill, J. (1984). El Utilitarismo. España. Editorial Alianza.

Mill, J. (1996). Principios de Economía Política. Brasil. Editora Nova Cultural.

Mishkin, F. (2007). “Consensos actuales en Política Monetaria: entre el arte y la ciencia” Cultura Económica. 35(70). pp. 44-65.

Mochón, F. (2006). Principios de Macroeconomía. España. Editorial Mc Graw Gill.

Navarrete, J. (2012). Política Económica. Primera Edición. México. Editorial Red Tercer Milenio.

Núñez, H. (1994). Finanzas Públicas. Argentina. Ediciones Macchi, p.15.

Rawls, J. (1995). Teoría De La Justicia. Segunda Edición. México. Editorial Fondo de Cultura Economía.

Tanzi, V. (1999). "La transición y la Transformación del papel del Estado". Finanzas \& Desarrollo. 36(2). pp. 20-23. 
Tanzi, V. (2010). "El Papel del Estado y la calidad del Sector Publico". Revista de la CEPAL. (71).pp.7-22. Disponible: http://www.cepal.org/es/publicaciones/37892-revista-la-cepal-no71. (Consultado 10/11/2018).

Urrunaga, R. y Otros. (2014). Fundamentos de Política Pública. Perú. Editorial Universidad del Pacifico Centro de Investigación.

Uvalle, R. (2002). “Los Fundamentos Institucionales de la Gestión Pública”. VII Congreso Internacional del CLAD sobre la Reforma del Estado y de la Administración Pública. Disponible:http://unpan1.un.org/intradoc/groups/public/documents/CLAD/clad0043511.pdf(Con sultado 15/11/2018).

Vargas, J. (2008). Perspectivas del Institucionalismo y el Neoinstitucionalismo. Revista Ciencia Administrativa. 3 (1). pp. 47-58.

Velasco, A. (1987). "Políticas de Estabilización y Teoría de Juegos" Colección Estudios Cieplan. 21(1). pp. 49.75. 